\title{
Eagle View: An Abstract Evaluation of Machine Learning Algorithms based on Data Properties
}

\author{
Dhairya Vyas \\ Research Scholer, \\ Computer Science and Engineering Department, \\ The Maharaja Sayajirao University Of Baroda, \\ Vadodara,Gujarat, India \\ dhairya.vyas-cse@msubaroda.ac.in
}

\author{
Viral V. Kapdia \\ Associate Professor, \\ Computer Science and Engineering Department, \\ The Maharaja Sayajirao University Of Baroda, \\ Vadodara, Gujarat, India \\ viral.kapadia-cse@msubaroda.ac.in
}

\begin{abstract}
Data can be generated from almost any type of information. Experimental Data Analysis (EDA) and Feature Engineering for machine learning models necessitate a thorough understanding of the different types of data. Algorithms that interpret and recall future data details use Machine Learning (ML) data. The majority of the data can be found on the internet. In terms of Machine Learning, the majority of the data can be grouped into four categories: numerical data, category data, time-series data, and text. Supervised Learning, a collection of unproven learning algorithms, is the subject of this research. Regression Models, Random Forests, Logistical Regressions, Support Vector Machines, Decision Trees, Neural Networks, Nave Bayes, t-SNE (t- Distributed Stochastic Neighborhood Embedding), k-means clustering, PCA (Principal Component Analysis), interim variance (TD), Q-Learning, and others are among the most recent machine learning approaches and other promising learning promises. Then concentrate on investigating and debating the issues with machine learning, as well as possible solutions. Investigate the Conflict Time and Learning Machine Release Effects on various data types after that. Finally, define data types based on what you've learned so far.
\end{abstract}

\section{Keywords- Datatypes; Supervised;} Reinforcement; Outliers; and Time Complexity

\section{INTRODUCTION}

Machine learning methods have helped progress in recent years as a result of the advent of big data. Machine learning is an AI branch that aims to analyses large quantities of data and simplify the automatic data reading system without relying on the system's apparent support. Machine learning algorithms are designed to find well-structured patterns in data that have never been seen before using various techniques, as well as to generate a more accurate prediction model than ever before. When previously unseen data is incorporated into a machine learning algorithm, the algorithm reads and forecasts the future based on previous feelings over time [2]. The power of machine learning is continuously being unleashed across a wide range of applications, including the Internet of Things (IoT), computer vision, natural language processing, speech processing, online complementary systems, Internet security, neuroscience, predictive forecasting, and fraud detection, to name a few [1].
Machine analysis of data learning uses time improving algorithms, so good data is needed to function correctly. These types [6]. You must first understand the data it uses to fully understand machinery learning. The research looks at which data types for machine learning are expected and where engineers may locate data sets that can be used in their study models.

Machine learning is a technology that evolves quickly and benefits companies and industry of all sizes. There are no limits to the number of applications [7]. Machine learning may help all types, from healthcare to financial services, transport to cyber safety and public marketing's, to respond and move forward quicker.

Machine data analysis uses time-consuming algorithms, so good information is necessary to work successfully for those types [1].

You must first understand the data it uses to fully understand machinery learning. Research can cover the type of data needed for the learning of a machine and which data sets are to be used in its machine learning model [6]. In addition, data sets can also be found. In order to make sure the model accurately interprets the data, data sets must first be incorporated into the machine learning algorithm, then verification (or test) data sets. Use the following data sets to further design your machine learning model after entering your training and verification sets into the system. If you make the system more information [9].

\section{TYPES OF DATA}

In order to understand what the database is, we need to talk data resources first. For example, an example is a line of data. A data set is a collection of instances with the same attribute for everyone. Machine learning models rely on four main data types that can be provided in various formats. This encompasses figures, categories, time and text data.

\section{A. Numerical Data}

Any measurement data, including height, weight or the cost of building your phone, is referred to as numerical data. Try to balance or sort numbers up or down to see if the data collection is numeric. Independent numbers (e.g., 26 students in a class) lie outside of the range, while constant numbers fall within one range (e.g., 3.6 percent interest rate) [12]. 


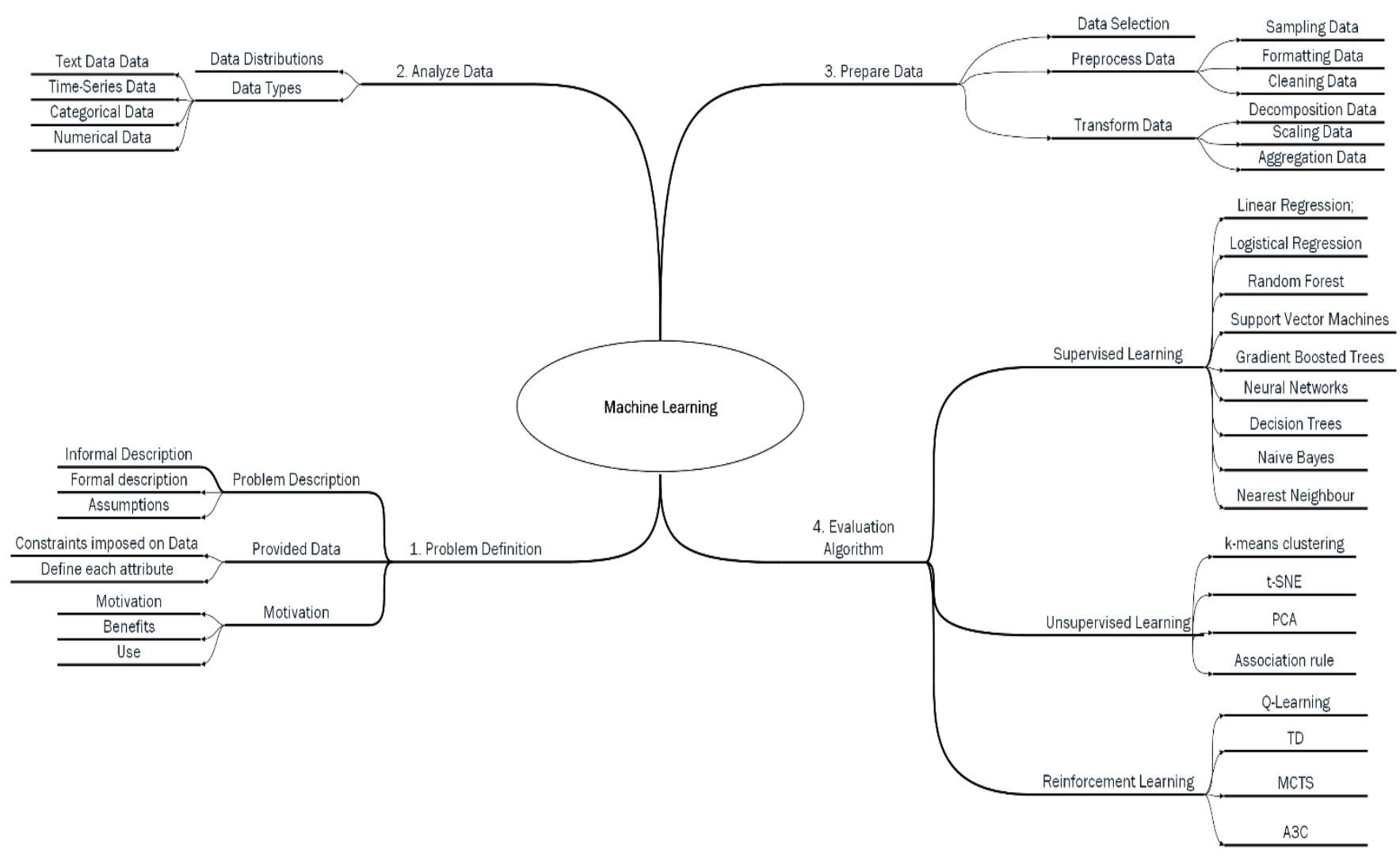

Fig. 1. Process of Machine Learning

\section{- $\quad$ Discrete Data}

It is usually calculated based on the number, such as: How often do I fill in "Heads"?

\section{- Continuous Data}

It has an infinite number of possibilities, for example, how much rain does it fall on a given day?

\section{B. Categorical Data}

The data are sorted by description in each category. A variety of other labelling may be used for sex, social class, race, home-country, companies [14]. Bear in mind that since there are no numbers for this type of data, it cannot be added, measured or sorted in any order. Data category is perfect for collecting people or thoughts with comparable features, making analyzing the data simpler for your model. Some numbers should be allocated to the category data, but they are not important for mathematics [16].

\section{- Qualitative Data}

Gender, Yes/No, Race, and so on.

\section{Time-series Data}

Data points and locations in timeline data are identified at specific times. Usually, this information is regularly collected. Reading and analyzing data for time series makes it easy to use any time-based metrics, from week to week, from month to month, from year to year. The only distinction between time series and numerical data is the predetermination of time series data for starting and end points, although numerical data are just a number collection and can depend on certain periods of time. A time series is a series of numbers taken over a period of time at regular intervals. Especially in certain areas such as finance, this is crucial.

\section{Text Data}

Text data are simply words, phrases or paragraphs that give a level of understanding to your learning models [11]. These phrases are often collected or analyzed by methods such as word frequency, text separation, or emotional analysis because they can be difficult for models to interpret. Mostly, you use fun activities, such as a word building bag, as the first thing you do with the text.

\section{MACHINE LEARNING TYPES}

The learning algorithm on the machine helps you to identify patterns from observed data, build models that explain the world and predict things, without having explicitly preprogrammed rules and models. Machine learning comes in many flavours, depending on the algorithm and its objectives. Machine learning algorithms may be grouped into three categories based on their purpose: supervised learning, uncontrolled learning, and enhanced learning.

\section{A. Supervised Learning}

The algorithm for machine learning permits the recognition of patterns in visual data, generation of models that depict the world, and the prediction of objects without strict rules and models. Machine learning can take several 
forms, depending on the algorithm and its objectives. Machines can be divided into three categories according to the intended application: supervised, supervised and enhanced reading $[11,4,16]$.

Controlled learning is one of the most basic kinds of machine learning. In this example, an algorithm for machine learning is trained on labelled data [23]. While the information needs to be labelled correctly in order to use this technique, supervised learning has great potential when used in the right circumstances.

A small training database is provided for the ML algorithm to work in supervised learning. The database is a small part of a bigger database that allows the algorithm to interpret the problem, solution, and data points [17]. The training database is similar in functionality to the final database and contains an algorithm that contains the label parameters required to solve the problem [22]. The algorithm then sets the cause and effect of the interaction of the variables in the database by identifying the relation between the respective parameters. At the end of training, the algorithm knows the functioning of the data and the relation between input and output. This solution is then supported by the final database, which is analysed in the same way as the training database. That means you will now learn new patterns and interactions as you train on new information after you have applied supervised machine learning skills [19].

- Linear Regression

- $\quad$ Support Vector Machines (SVM)

- Logistical Regression

- Naive Bayes

- Random Forest

- Nearest Neighbour

- Gradient Boosted Trees

- Decision Trees

- Neural Networks

\section{B. Unsupervised learning}

Unsupported machine learning should work with undeclared statistics. This makes it impossible to manage big datasets in the data base system to read the machine [12]. Labels allow the algorithm to determine the exact nature of the connection in supervised reading between any two data points.

\section{QuAnTiTATIVE ANALYSIS}

In this section the various types of data Numerical Data, category data, Time Series data, and Textual Data are analyzed by Complexity and Accuracy parameters. There are a number of places where you can get electronic learning data,
However, unchecked reading lacks labels to work with which hidden structures are created [19]. In an incomprehensible way, the algorithm realizes the connection between data points without the need for human input. The development of these secret structures transforms unchecked reading skills. Unattended readability can respond to data by modifying hidden properties drastically rather than by defining and specifying a problem statement. This enables learning algorithms to improve even more after correction [21].

- $\quad \mathrm{k}-\mathrm{means}$ clustering

- t-SNE (t-Distributed Stochastic Neighbor Embedding)

- PCA (Principal Component Analysis)

- Association rule

\section{Reinforcement learning}

Increasing education is influenced directly by how people learn in everyday lives from data [14]. It involves an algorithm for trial and error which improves itself and learns from new situations. Positive results will be rewarded or "reinforced," while negative results will be prevented or "punished." Intensified learning is based on the psychological idea of conditioning by putting the algorithm in a working environment based on an interpreter and a reward system. Each interpreter chooses if the output results are favourable for every iteration of the algorithm [13]. If the programme's solution is found the correct one, the translator improves the algorithm. When the result is disadvantageous, the algorithm must repeat the process until the results are best discovered. The rewards system is in the vast majority of cases proportionate directly to the effectiveness of the result [15].

In classical enhancement learning situations, the solution is not of absolute value, like finding the shortest way to find two points on a map [16]. Instead, a percentage value is assigned to the productivity score. The higher the value, the more reward the algorithm is. In return for the best possible reward, the programme was therefore planned to offer the best possible solution [21].

- $\quad$ Temporal Difference (TD)

- Asynchronous Actor-Critic Agents (A3C)

- Monte-Carlo Tree Search (MCTS)

- Q-Learning

five of the most used ML dataset resources are Google, Microsoft, AWS, UCI and Data.gov.

In all tables, conflict analysis was done when $\mathrm{n}=$ data point and $\mathrm{p}=$ parameters. 


\section{A. Numerical Data}

In this part we have taken three datasets Iris, Wine and Cancer for analysis of different ML approaches among then we can see that Logistical regression model give best performance.

TABLE I. NUMERICAL DATA ANALYSIS

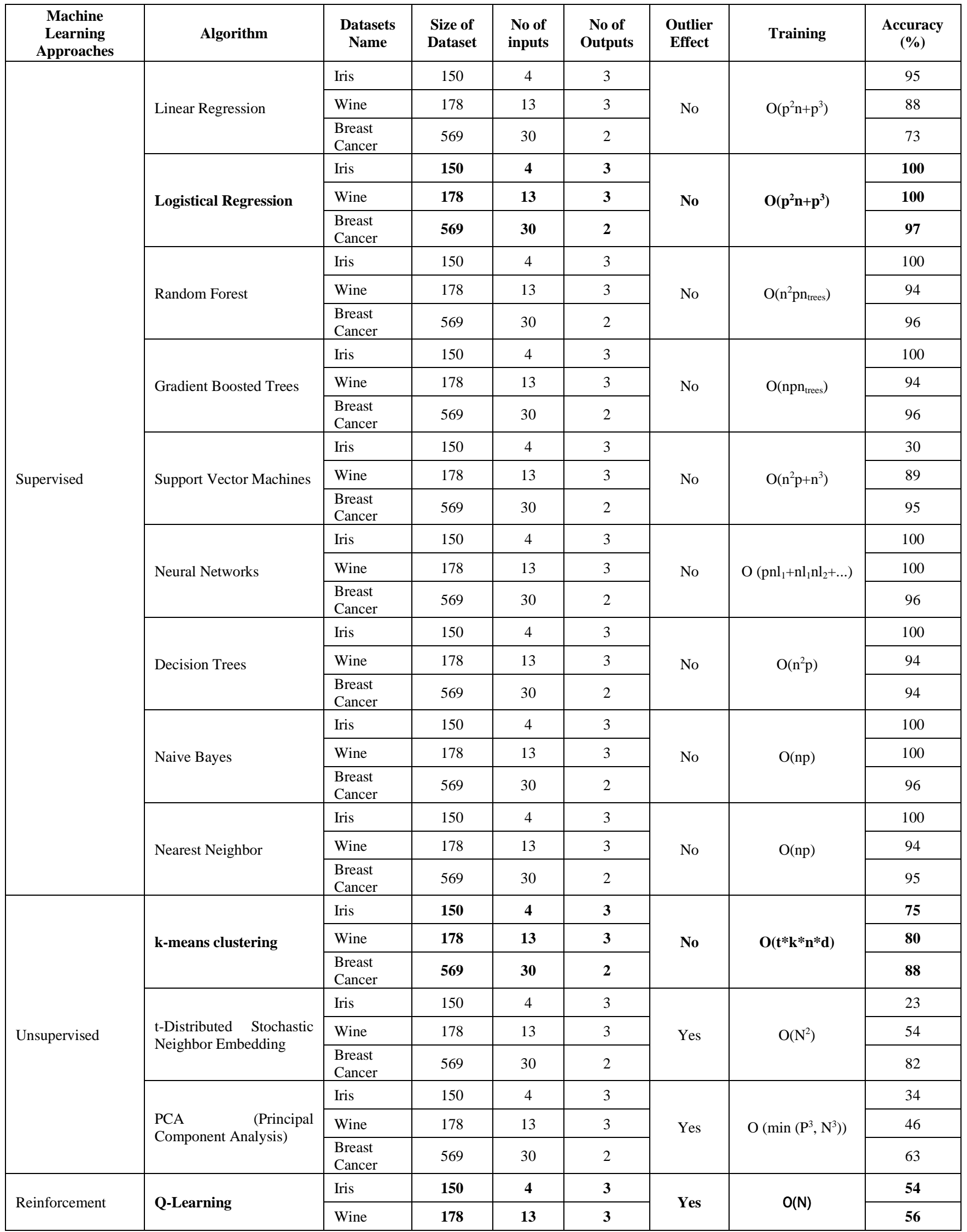




\begin{tabular}{|c|c|c|c|c|c|c|c|}
\hline & $\begin{array}{l}\text { Breast } \\
\text { Cancer }\end{array}$ & 569 & 30 & 2 & & & 53 \\
\hline \multirow{3}{*}{$\begin{array}{l}\text { Temporal } \\
\text { (TD) }\end{array}$} & Iris & 150 & 4 & 3 & \multirow{3}{*}{ Yes } & \multirow{3}{*}{$\mathrm{O}\left(\mathrm{N}^{3}\right)$} & 64 \\
\hline & Wine & 178 & 13 & 3 & & & 66 \\
\hline & $\begin{array}{l}\text { Breast } \\
\text { Cancer }\end{array}$ & 569 & 30 & 2 & & & 63 \\
\hline \multirow{3}{*}{$\begin{array}{l}\text { Monte-Carlo Tree Search } \\
\text { (MCTS) }\end{array}$} & Iris & 150 & 4 & 3 & \multirow{3}{*}{ Yes } & \multirow{3}{*}{$\mathrm{O}\left(\mathrm{N}^{3}\right)$} & 55 \\
\hline & Wine & 178 & 13 & 3 & & & 54 \\
\hline & $\begin{array}{l}\text { Breast } \\
\text { Cancer }\end{array}$ & 569 & 30 & 2 & & & 57 \\
\hline \multirow{3}{*}{$\begin{array}{l}\text { Asynchronous Actor- } \\
\text { Critic Agents (A3C) }\end{array}$} & Iris & 150 & 4 & 3 & \multirow{3}{*}{ Yes } & \multirow{3}{*}{$\mathrm{O}(\mathrm{N})$} & 57 \\
\hline & Wine & 178 & 13 & 3 & & & 66 \\
\hline & $\begin{array}{l}\text { Breast } \\
\text { Cancer }\end{array}$ & 569 & 30 & 2 & & & 68 \\
\hline
\end{tabular}

\section{B. Categorical Data}

In this part we have taken three datasets car, toy and Mushroom for analysis of different ML approaches among then we can see that Decision Tree model give best performance.

TABLE II. CATEGORICAL DATA ANALYSIS

\begin{tabular}{|c|c|c|c|c|c|c|c|c|}
\hline $\begin{array}{c}\text { Machine } \\
\text { Learning } \\
\text { Approaches }\end{array}$ & Algorithm & $\begin{array}{l}\text { Dataset } \\
\text { Name }\end{array}$ & $\begin{array}{l}\text { Size of } \\
\text { Dataset }\end{array}$ & $\begin{array}{l}\text { No of } \\
\text { inputs }\end{array}$ & $\begin{array}{c}\text { No of } \\
\text { Outputs }\end{array}$ & $\begin{array}{c}\text { Outlier } \\
\text { Effect }\end{array}$ & $\begin{array}{c}\text { Time } \\
\text { Complexity }\end{array}$ & Accuracy \\
\hline \multirow{27}{*}{ Supervised } & \multirow{3}{*}{ Linear Regression } & Car & 1728 & 6 & 4 & \multirow{3}{*}{ Yes } & \multirow{3}{*}{$\mathrm{O}\left(\mathrm{p}^{2} n+\mathrm{p}^{3}\right)$} & 32 \\
\hline & & Toy & 20 & 4 & 2 & & & 51 \\
\hline & & Mushroom & 8124 & 22 & 2 & & & 75 \\
\hline & \multirow{3}{*}{ Logistical Regression } & Car & 1728 & 6 & 4 & \multirow{3}{*}{ No } & \multirow{3}{*}{$O\left(p^{2} n+p^{3}\right)$} & 74 \\
\hline & & Toy & 20 & 4 & 2 & & & 100 \\
\hline & & Mushroom & 8124 & 22 & 2 & & & 94 \\
\hline & \multirow{3}{*}{ Random Forest } & Car & 1728 & 6 & 4 & \multirow{3}{*}{ Yes } & \multirow{3}{*}{$\mathrm{O}\left(\mathrm{n}^{2} \mathrm{pn}\right.$ trees $)$} & 68 \\
\hline & & Toy & 20 & 4 & 2 & & & 100 \\
\hline & & Mushroom & 8124 & 22 & 2 & & & 89 \\
\hline & \multirow{3}{*}{ Gradient Boosted Trees } & Car & 1728 & 6 & 4 & \multirow{3}{*}{ No } & \multirow{3}{*}{$\mathrm{O}\left(\mathrm{npn}_{\text {trees }}\right)$} & 97 \\
\hline & & Toy & 20 & 4 & 2 & & & 100 \\
\hline & & Mushroom & 8124 & 22 & 2 & & & 100 \\
\hline & \multirow{3}{*}{ Support Vector Machines } & Car & 1728 & 6 & 4 & \multirow{3}{*}{ Yes } & \multirow{3}{*}{$\mathrm{O}\left(\mathrm{n}^{2} \mathrm{p}+\mathrm{n}^{3}\right)$} & 68 \\
\hline & & Toy & 20 & 4 & 2 & & & 25 \\
\hline & & Mushroom & 8124 & 22 & 2 & & & 91 \\
\hline & \multirow{3}{*}{ Neural Networks } & Car & 1728 & 6 & 4 & \multirow{3}{*}{ No } & \multirow{3}{*}{$\mathrm{O}\left(\mathrm{pnl}_{1}+\mathrm{nl}_{1} \mathrm{nl}_{2}+\ldots\right)$} & 90 \\
\hline & & Toy & 20 & 4 & 2 & & & 100 \\
\hline & & Mushroom & 8124 & 22 & 2 & & & 100 \\
\hline & \multirow{3}{*}{ Decision Trees } & Car & 1728 & 6 & 4 & \multirow{3}{*}{ No } & \multirow{3}{*}{$\mathbf{O}\left(\mathbf{n}^{2} \mathbf{p}\right)$} & 96 \\
\hline & & Toy & 20 & 4 & 2 & & & 100 \\
\hline & & Mushroom & 8124 & 22 & 2 & & & 100 \\
\hline & \multirow{3}{*}{ Naive Bayes } & Car & 1728 & 6 & 4 & \multirow{3}{*}{ Yes } & \multirow{3}{*}{$\mathrm{O}(\mathrm{np})$} & 59 \\
\hline & & Toy & 20 & 4 & 2 & & & 100 \\
\hline & & Mushroom & 8124 & 22 & 2 & & & 91 \\
\hline & \multirow{3}{*}{ Nearest Neighbor } & Car & 1728 & 6 & 4 & \multirow{3}{*}{ No } & \multirow{3}{*}{$\mathrm{O}(\mathrm{np})$} & 83 \\
\hline & & Toy & 20 & 4 & 2 & & & 100 \\
\hline & & Mushroom & 8124 & 22 & 2 & & & 100 \\
\hline \multirow{2}{*}{ Unsupervised } & \multirow{2}{*}{ k-means clustering } & Car & 1728 & 6 & 4 & No & ar & 55 \\
\hline & & Toy & 20 & 4 & 2 & 100 & 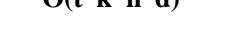 & 72 \\
\hline
\end{tabular}




\begin{tabular}{|c|c|c|c|c|c|c|c|c|}
\hline & & Mushroom & 8124 & 22 & 2 & & & 100 \\
\hline & \multirow{3}{*}{$\begin{array}{l}\text { t-Distributed Stochastic } \\
\text { Neighbor Embedding }\end{array}$} & Car & 1728 & 6 & 4 & \multirow{3}{*}{ Yes } & \multirow{3}{*}{$\mathrm{O}\left(\mathrm{N}^{2}\right)$} & 45 \\
\hline & & Toy & 20 & 4 & 2 & & & 44 \\
\hline & & Mushroom & 8124 & 22 & 2 & & & 60 \\
\hline & \multirow{3}{*}{$\begin{array}{l}\text { PCA (Principal } \\
\text { Component Analysis) }\end{array}$} & Car & 1728 & 6 & 4 & \multirow{3}{*}{ Yes } & \multirow{3}{*}{$\mathrm{O}\left(\min \left(\mathrm{P}^{3}, \mathrm{~N}^{3}\right)\right)$} & 55 \\
\hline & & Toy & 20 & 4 & 2 & & & 54 \\
\hline & & Mushroom & 8124 & 22 & 2 & & & 50 \\
\hline \multirow{12}{*}{ Reinforcement } & \multirow{3}{*}{ Q-Learning } & Car & 1728 & 6 & 4 & \multirow{3}{*}{ Yes } & \multirow{3}{*}{$\mathrm{O}\left(\mathrm{N}^{3}\right)$} & 64 \\
\hline & & Toy & 20 & 4 & 2 & & & 66 \\
\hline & & Mushroom & 8124 & 22 & 2 & & & 63 \\
\hline & & Car & 1728 & 6 & 4 & & & \\
\hline & $\begin{array}{l}\text { lemporal Dilterence } \\
\text { (TD) }\end{array}$ & Toy & 20 & 4 & 2 & Yes & - & - \\
\hline & & Mushroom & 8124 & 22 & 2 & & & \\
\hline & & Car & 1728 & 6 & 4 & & & \\
\hline & $\begin{array}{l}\text { Monte-Carlo Tree Search } \\
\text { (MCTS) }\end{array}$ & Toy & 20 & 4 & 2 & Yes & - & - \\
\hline & & Mushroom & 8124 & 22 & 2 & & & \\
\hline & & Car & 1728 & 6 & 4 & & & \\
\hline & $\begin{array}{l}\text { Asynchronous Actor- } \\
\text { Critic Agents (A3C) }\end{array}$ & Toy & 20 & 4 & 2 & Yes & - & - \\
\hline & & Mushroom & 8124 & 22 & 2 & & & \\
\hline
\end{tabular}

\section{Time-Series Data}

In this part we have taken three datasets Flight for analysis of different ML approaches among then we can see that Decision tree model give best performance.

TABLE III. TIME-SERIES DATA ANALYSIS

\begin{tabular}{|c|c|c|c|c|c|c|c|c|}
\hline $\begin{array}{c}\text { Machine } \\
\text { Learning } \\
\text { Approaches }\end{array}$ & Algorithm & $\begin{array}{c}\text { Dataset } \\
\text { Name }\end{array}$ & $\begin{array}{l}\text { Size of } \\
\text { Dataset }\end{array}$ & $\begin{array}{c}\text { No of } \\
\text { inputs }\end{array}$ & $\begin{array}{l}\text { No of } \\
\text { Outputs }\end{array}$ & $\begin{array}{c}\text { Outlier } \\
\text { Effect }\end{array}$ & $\begin{array}{c}\text { Time } \\
\text { Complexity } \\
\end{array}$ & Accuracy \\
\hline \multirow{9}{*}{ Supervised } & Linear Regression & Flight & 583986 & 4 & 2 & No & $\mathrm{O}\left(\mathrm{p}^{2} \mathrm{n}+\mathrm{p}^{3}\right)$ & 61 \\
\hline & Logistical Regression & Flight & 583986 & 4 & 2 & No & $O\left(p^{2} n+p^{3}\right)$ & 81 \\
\hline & Random Forest & Flight & 583986 & 4 & 2 & No & $\mathrm{O}\left(\mathrm{n}^{2} \mathrm{pn}_{\text {trees }}\right)$ & 81 \\
\hline & Gradient Boosted Trees & Flight & 583986 & 4 & 2 & No & $\mathrm{O}\left(\mathrm{npn}_{\text {trees }}\right)$ & 84 \\
\hline & Support Vector Machines & Flight & 583986 & 4 & 2 & Yes & $\mathrm{O}\left(\mathrm{n}^{2} \mathrm{p}+\mathrm{n}^{3}\right)$ & 19 \\
\hline & Neural Networks & Flight & 583986 & 4 & 2 & No & $\begin{array}{c}\mathrm{O} \\
\left(\mathrm{pnl}_{1}+\mathrm{nl}_{1} \mathrm{nl}_{2}+\ldots\right)\end{array}$ & 81 \\
\hline & Decision Trees & Flight & 583986 & 4 & 2 & No & $\mathbf{O}\left(\mathbf{n}^{2} \mathbf{p}\right)$ & 90 \\
\hline & Naive Bayes & Flight & 583986 & 4 & 2 & No & $\mathrm{O}(\mathrm{np})$ & 81 \\
\hline & Nearest Neighbor & Flight & 583986 & 4 & 2 & No & $\mathrm{O}(\mathrm{np})$ & 86 \\
\hline \multirow{3}{*}{ Unsupervised } & k-means clustering & Flight & 583986 & 4 & 2 & Yes & $\mathrm{O}(\mathrm{t} * \mathrm{k} * \mathrm{n} * \mathrm{~d})$ & 52 \\
\hline & $\begin{array}{l}\text { t-Distributed Stochastic } \\
\text { Neighbor Embedding }\end{array}$ & Flight & 583986 & 4 & 2 & Yes & $\mathbf{O}\left(\mathbf{N}^{2}\right)$ & 58 \\
\hline & $\begin{array}{l}\text { PCA (Principal Component } \\
\text { Analysis) }\end{array}$ & Flight & 583986 & 4 & 2 & Yes & $\begin{array}{c}\mathrm{O}\left(\min \left(\mathrm{P}^{3}\right.\right. \\
\left.\left.\mathrm{N}^{3}\right)\right)\end{array}$ & 54 \\
\hline \multirow{4}{*}{ Reinforcement } & Q-Learning & Flight & 583986 & 4 & 2 & Yes & $\mathrm{O}(\mathrm{N})$ & 90 \\
\hline & Temporal Difference (TD) & Flight & 583986 & 4 & 2 & Yes & $\mathrm{O}\left(\mathrm{N}^{3}\right)$ & 65 \\
\hline & $\begin{array}{lll}\begin{array}{l}\text { Monte-Carlo } \\
\text { (MCTS) }\end{array} & \text { Tree } & \text { Search } \\
\end{array}$ & Flight & 583986 & 4 & 2 & Yes & $\mathrm{O}\left(\mathrm{N}^{3}\right)$ & 60 \\
\hline & $\begin{array}{ll}\text { Asynchronous } & \text { Actor-Critic } \\
\text { Agents (A3C) } & \\
\end{array}$ & Flight & 583986 & 4 & 2 & Yes & $\mathrm{O}(\mathrm{N})$ & 65 \\
\hline
\end{tabular}




\section{Text Data}

In this part we have taken three datasets Yelp, amazon and IMDB for analysis of different ML approaches among then we can see that Neural Networks model give best performance.

TABLE IV. TEXT DATA ANALYSIS

\begin{tabular}{|c|c|c|c|c|c|c|c|c|}
\hline $\begin{array}{c}\text { Machine } \\
\text { Learning } \\
\text { Approaches }\end{array}$ & Algorithm & $\begin{array}{l}\text { Dataset } \\
\text { Name }\end{array}$ & $\begin{array}{l}\text { Size of } \\
\text { Dataset }\end{array}$ & $\begin{array}{l}\text { No of } \\
\text { inputs }\end{array}$ & $\begin{array}{c}\text { No of } \\
\text { Outputs }\end{array}$ & $\begin{array}{l}\text { Outlier } \\
\text { Effect }\end{array}$ & $\begin{array}{c}\text { Time } \\
\text { Complexity }\end{array}$ & Accuracy \\
\hline & \multirow{3}{*}{ Linear Regression } & Yelp & 1000 & \multirow{3}{*}{ Text } & \multirow{3}{*}{2} & \multirow{3}{*}{ No } & \multirow{3}{*}{$O\left(p^{2} n+p^{3}\right)$} & 60 \\
\hline & & Amazon & 1000 & & & & & 65 \\
\hline & & IMDB & 1000 & & & & & 64 \\
\hline & \multirow{3}{*}{ Logistical Regression } & Yelp & 1000 & \multirow{3}{*}{ Text } & \multirow{3}{*}{2} & \multirow{3}{*}{ No } & \multirow{3}{*}{$O\left(p^{2} n+p^{3}\right)$} & 79 \\
\hline & & Amazon & 1000 & & & & & 80 \\
\hline & & IMDB & 1000 & & & & & 79 \\
\hline & \multirow{3}{*}{ Random Forest } & Yelp & 1000 & \multirow{3}{*}{ Text } & \multirow{3}{*}{2} & \multirow{3}{*}{ No } & \multirow{3}{*}{$\mathrm{O}\left(\mathrm{n}^{2} \mathrm{pn}_{\text {trees }}\right)$} & 56 \\
\hline & & Amazon & 1000 & & & & & 56 \\
\hline & & IMDB & 1000 & & & & & 53 \\
\hline & \multirow{3}{*}{ Gradient Boosted Trees } & Yelp & 1000 & \multirow{3}{*}{ Text } & \multirow{3}{*}{2} & \multirow{3}{*}{ No } & \multirow{3}{*}{$\mathrm{O}\left(\mathrm{npn}_{\text {trees }}\right)$} & 77 \\
\hline & & Amazon & 1000 & & & & & 81 \\
\hline & & IMDB & 1000 & & & & & 67 \\
\hline & & Yelp & 1000 & & & & & 48 \\
\hline & Support Vector Machines & Amazon & 1000 & Text & 2 & No & $\mathrm{O}\left(\mathrm{n}^{2} \mathrm{p}+\mathrm{n}^{3}\right)$ & 49 \\
\hline & & IMDB & 1000 & & & & & 49 \\
\hline & & Yelp & 1000 & & & & & 81 \\
\hline & Neural Networks & Amazon & 1000 & Text & 2 & No & $\frac{\text { O }}{\left(\mathrm{nnl}_{1}+\mathbf{n} \mathrm{ll}_{\mathrm{t}}\right)}$ & 77 \\
\hline & & IMDB & 1000 & & & & & 83 \\
\hline & & Yelp & 1000 & & & & & 76 \\
\hline & Decision Trees & Amazon & 1000 & Text & 2 & No & $\mathrm{O}\left(\mathrm{n}^{2} \mathrm{p}\right)$ & 77 \\
\hline & & IMDB & 1000 & & & & & 67 \\
\hline & & Yelp & 1000 & & & & & 78 \\
\hline & Naive Bayes & Amazon & 1000 & Text & 2 & No & $\mathrm{O}(\mathrm{np})$ & 78 \\
\hline & & IMDB & 1000 & & & & & 79 \\
\hline & & Yelp & 1000 & & & & & 66 \\
\hline & Nearest Neighbor & Amazon & 1000 & Text & 2 & No & $\mathrm{O}(\mathrm{np})$ & 62 \\
\hline & & IMDB & 1000 & & & & & 59 \\
\hline & & Yelp & 1000 & & & & & 65 \\
\hline & k-means clustering & Amazon & 1000 & Text & 2 & No & $\mathbf{O}\left(\mathbf{t}^{*} \mathbf{k} * \mathbf{n} * \mathbf{d}\right)$ & 69 \\
\hline & & IMDB & 1000 & & & & & 59 \\
\hline & & Yelp & 1000 & & & & & \\
\hline Unsupervised & $\begin{array}{l}\text { t-Distributed Stochastic } \\
\text { Neighbor Embedding }\end{array}$ & Amazon & 1000 & Text & 2 & Yes & - & - \\
\hline & & IMDB & 1000 & & & & & \\
\hline & & Yelp & 1000 & & & & & \\
\hline & $\begin{array}{l}\text { PCA (Principal } \\
\text { Component Analysis) }\end{array}$ & Amazon & 1000 & Text & 2 & Yes & - & - \\
\hline & & IMDB & 1000 & & & & & \\
\hline & & Yelp & 1000 & & & & & 54 \\
\hline & Q-Learning & Amazon & 1000 & Text & 2 & Yes & $\mathrm{O}(\mathrm{N})$ & 56 \\
\hline & & IMDB & 1000 & & & & & 53 \\
\hline & & Yelp & 1000 & & & & & 64 \\
\hline & $\begin{array}{l}\text { Temporal Difference } \\
\text { (TD) }\end{array}$ & Amazon & 1000 & Text & 2 & Yes & $\mathrm{O}\left(\mathrm{N}^{3}\right)$ & 66 \\
\hline Reinforcement & & IMDB & 1000 & & & & & 63 \\
\hline & & Yelp & 1000 & & & & & 55 \\
\hline & $\begin{array}{l}\text { Monte-Carlo Tree Search } \\
\text { (MCTS) }\end{array}$ & Amazon & 1000 & Text & 2 & Yes & $\mathrm{O}\left(\mathrm{N}^{3}\right)$ & 54 \\
\hline & & IMDB & 1000 & & & & & 57 \\
\hline & & Yelp & 1000 & & & & & 57 \\
\hline & $\begin{array}{l}\text { Asynchronous Actor- } \\
\text { Critic Agents (A3C) }\end{array}$ & Amazon & 1000 & Text & 2 & Yes & $\mathrm{O}(\mathrm{N})$ & 66 \\
\hline & & IMDB & 1000 & & & & & 68 \\
\hline
\end{tabular}




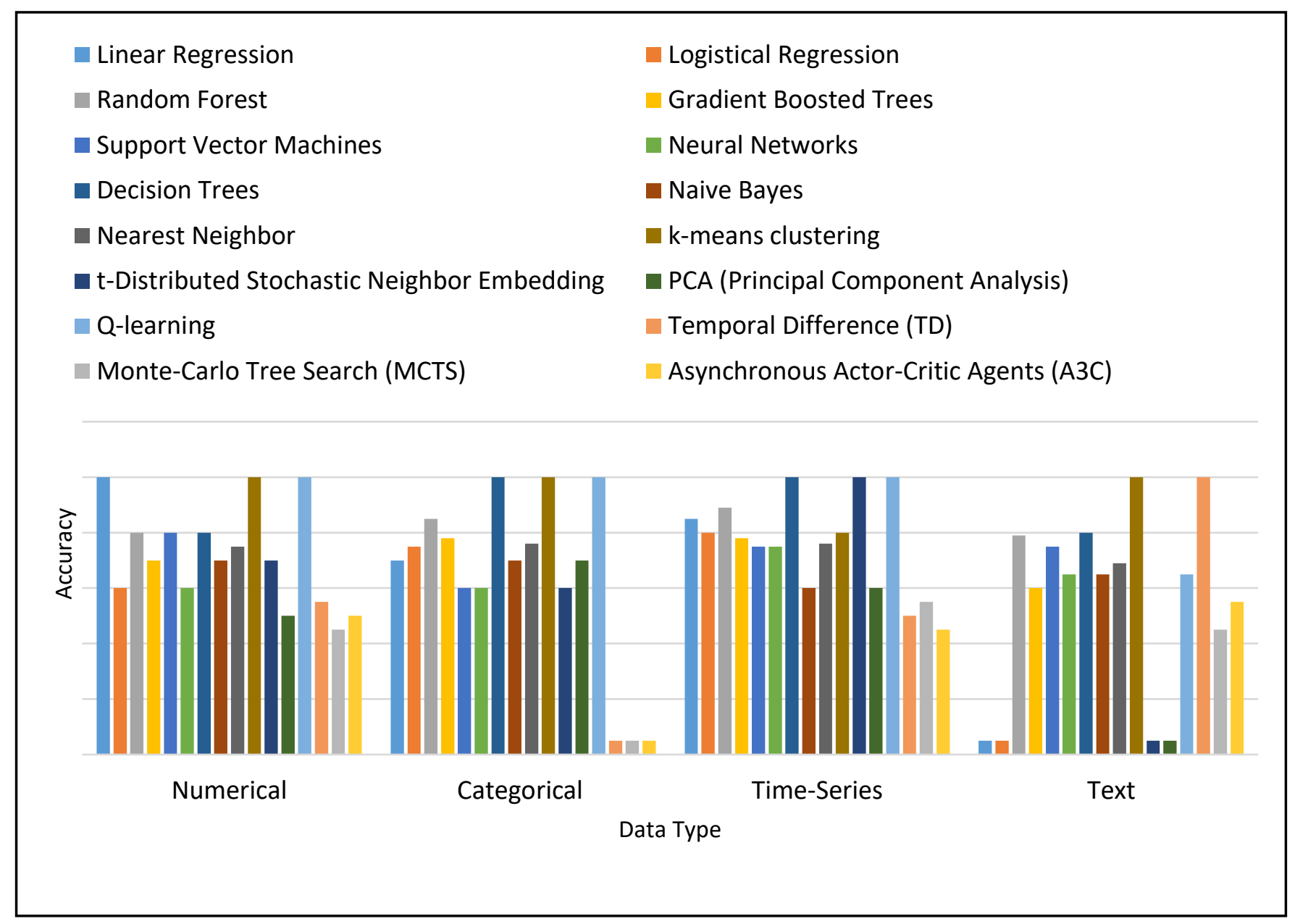

Fig. 2. Explainability-Accuracy Tradeoff

\section{SUMMARY}

As from the tables of analysis it can be conclude that Numerical data are perform batter for Linear Regression supervise learning, K-Nearest Neighbor for unsupervised learning and Q-learning for Reinforcement learning. Categorical data are performed batter for Decision Tree supervise learning, K-Nearest Neighbor for unsupervised learning and Q-learning for Reinforcement learning. TimeSeries data are performed batter for Decision tree supervise learning, t-DSNE for unsupervised learning and Q-learning for Reinforcement learning. Textual data are performed batter for Neural Network supervise learning, KNN for unsupervised learning and Temporal Difference for Reinforcement learning.

In a dynamic environment, the machine learning algorithm can handle multi-featured data. Despite its benefits, there are still difficulties and difficulties. To predict the large number of new classes, another approach is required of the machine learning algorithm. Additionally, the machine learning algorithm's improved analytical skills posed new difficulties and challenges when it came to maintaining privacy. A lack of well-defined raw data, leading to improper findings, complicates the learning process. In addition, human experts are necessary to ensure the correct use of the algorithm for machine learning. Interpreting the results is a big challenge in the machine learning algorithm.
Machine learning technology reveals secret learning details among a slew of big data bullets. However, problems such as large-size distribution of computers, distribution, versatility and data streaming are plaguing it. Data duplication also has an important impact on algorithms for machine learning. In many situations it is impossible to classify large quantities of dynamic data. The big problem with algorithms for machine learning is they are susceptible to errors. Machine learning techniques are now different. The learning algorithm takes accurate data for future forecasts. Finding a range of time consuming and resource-intensive concerns requires a massive amount of data.

\section{REFERENCES}

[1] Rasyid, L. A., \&amp; Andayani, S. (2018). Review on clustering algorithms based on data type: Towards the method for data combined of numeric-fuzzy linguistics. Journal of Physics: Conference Series, 1097, 012082. doi:10.1088/1742-6596/1097/1/012082.

[2] J. Qiu, Q. Wu, G. Ding, Y. Xu, and S. Feng, "A survey of machine learning for big data processing," EURASIP J. Adv. Signal Process., vol. 2016, no. 1, 2016, doi: 10.1186/s13634-016-0355-x.

[3] P. Paokanta, M. Ceccarelli, and S. Srichairatanakool, "The effeciency of data types for classification performance of machine learning techniques for screening $\beta$-Thalassemia," 2010 3rd Int. Symp. Appl. Sci. Biomed. Commun. Technol. ISABEL 2010, pp. 1-4, 2010, doi: 10.1109/ISABEL.2010.5702769.

[4] U. Narayanan, A. Unnikrishnan, V. Paul, and S. Joseph, "A survey on various supervised classification algorithms," 2017 Int. Conf. Energy, 
Commun. Data Anal. Soft Comput. ICECDS 2017, pp. 2118-2124, 2018, doi: 10.1109/ICECDS.2017.8389824.

[5] N. Thomas Rincy and R. Gupta, "A Survey on Machine Learning Approaches and Its Techniques:," 2020 IEEE Int. Students' Conf. Electr. Electron. Comput. Sci. SCEECS 2020, 2020, doi: 10.1109/SCEECS48394.2020.190.

[6] A. Dhillon and A. Singh, "Machine Learning in Healthcare Data Analysis: A Survey," J. Biol. Today's World, vol. 8, no. 6, pp. 1-10, 2019, doi: 10.15412/J.JBTW.01070206.

[7] J. Latif, C. Xiao, A. Imran, and S. Tu, "Medical imaging using machine learning and deep learning algorithms: A review," 2019 2nd Int. Conf. Comput. Math. Eng. Technol. iCoMET 2019, pp. 1-5, 2019, doi: 10.1109/ICOMET.2019.8673502.

[8] S. L. Problem and F. Accommodation, "Machine Lear," pp. 783-821.

[9] P. Verma and R. Kumar, "A Literature Survey on Classification Algorithms of Machine Learning," Int. J. Comput. Appl., vol. 179, no. 53, pp. 47-50, 2018, doi: 10.5120/ijca2018917378.

[10] S. R. Guruvayur and R. Suchithra, "A detailed study on machine learning techniques for data mining," Proc. - Int. Conf. Trends Electron. Informatics, ICEI 2017, vol. 2018-January, pp. 1187-1192, 2018, doi: 10.1109/ICOEI.2017.8300900.

[11] M. Vennapusa and S. Bhyrapuneni, "A comprehensive study of machine learning mechanisms on big data," Int. J. Recent Technol. Eng., vol. 7, no. 6, pp. 773-779, 2019.

[12] S. Datta and A. Arputharaj, "An Analysis of Several Machine Learning Algorithms for Imbalanced Classes," 5th Int. Conf. Soft Comput. Mach. Intell. ISCMI 2018, pp. 22-27, 2018, doi: 10.1109/ISCMI.2018.8703244.

[13] P. S. Helode, Dr. K. H. Walse, and Karande M.U., "An Online Secure Social Networking with Friend Discovery System," Int. J. Innov. Res. Comput. Commun. Eng., vol. 5, no. 4, pp. 8198-8205, 2017, doi: 10.15680/IJIRCCE.2017.

[14] S. A. Ebrahim, J. Poshtan, S. M. Jamali, and N. A. Ebrahim, "Quantitative and Qualitative Analysis of Time-Series Classification Using Deep Learning," IEEE Access, vol. 8, pp. 90202-90215, 2020, doi: 10.1109/ACCESS.2020.2993538.

[15] I. Khan, X. Zhang, M. Rehman, and R. Ali, "A Literature Survey and Empirical Study of Meta-Learning for Classifier Selection," IEEE Access, vol. 8, pp. 10262-10281, 2020, doi: 10.1109/ACCESS.2020.2964726.

[16] S. Ray, "A Quick Review of Machine Learning Algorithms," Proc. Int. Conf. Mach. Learn. Big Data, Cloud Parallel Comput. Trends, Prespectives Prospect. Com. 2019, pp. 35-39, 2019, doi: 10.1109/COMITCon.2019.8862451.

[17] M. Binkhonain and L. Zhao, "A review of machine learning algorithms for identification and classification of non-functional requirements," Expert Syst. with Appl. X, vol. 1, 2019, doi: 10.1016/j.eswax.2019.100001.

[18] Chakure, A. (2019, August 5). Introduction to machine learning and different types of machine learning algorithms. Hacker Noon. https://hackernoon.com/introduction-to-machine-learning-anddifferent-types-of-machine-learning-algorithms-3z57i242u

[19] Commonly used machine learning algorithms (with Python and R codes). (2020, April 15). Analytics Vidhya. https://www.analyticsvidhya.com/blog/2017/09/common-machinelearning-algorithms/

[20] Heidenreich, H. (2018, December 4). What are the types of machine learning? Medium. https://towardsdatascience.com/what-are-thetypes-of-machine-learning-e2b9e5d1756f

[21] Machine learning algorithms: 4 types you should know. (2018, December 7). Best Web \& Mobile App Developers Company | The APP Solutions. https://theappsolutions.com/blog/development/machine-learningalgorithm-types/

[22] Machine learning algorithms: A comprehensive guide. (2021, February 25). Professional Certification Courses, Classroom And Virtual Trainings. https://www.knowledgehut.com/blog/datascience/machine-learning-algorithms

[23] Zhang, A. (2020, August 20). Data types from a machine learning perspective with examples. Medium. https://towardsdatascience.com/data-types-from-a-machine-learningperspective-with-examples-111ac679e8bc 